\title{
Measurement of gene amplifications related to drug resistance in Plasmodium falciparum using droplet digital PCR
}

\author{
Suttipat Srisutham¹, Kanokon Suwannasin², Rungniran Sugaram³ ${ }^{3}$ Arjen M. Dondorp ${ }^{2,4}$ \\ and Mallika Imwong ${ }^{2,5^{*}}$ (])
}

\begin{abstract}
Background: Copy number variations (CNVs) of the Plasmodium falciparum multidrug resistance 1 (pfmdr 1 ), P. falciparum plasmepsin2 (pfplasmepsin2) and P. falciparum GTP cyclohydrolase 1 (pfgch1) genes are associated with anti-malarial drug resistance in P. falciparum malaria. Droplet digital PCR (ddPCR) assays have been developed for accurate assessment of CNVs in several human genes. The aim of the present study was to develop and validate ddPCR assays for detection of the CNVs of P. falciparum genes associated with resistance to anti-malarial drugs.

Methods: A multiplex ddPCR assay was developed to detect the CNVs in the pfmdr1 and pfplasmepsin2 genes, while a duplex ddPCR assay was developed to detect CNV in the pfgch1 gene. The gene copy number (GCN) quantification limit, as well as the accuracy and precision of the ddPCR assays were determined and compared to conventional quantitative PCR (qPCR). In order to reduce the cost of testing, a multiplex ddPCR assay of two target genes, pfmdr 1 and pfplasmepsin2, was validated. In addition, the CNVs of genes of field samples collected from Thailand from 2015 to $2019(n=84)$ were assessed by ddPCR and results were compared to qPCR as the reference assay.

Results: There were no significant differences between the GCN results obtained from uniplex and multiplex ddPCR assays for detection of CNVs in the pfmdr1 and pfplasmepsin 2 genes ( $p=0.363$ and 0.330 , respectively). Based on the obtained gene copy number quantification limit, the accuracy and percent relative standard deviation (\%RSD) value of the multiplex ddPCR assay were $95 \%$ and 5\%, respectively, for detection of the CNV of the pfmdr 1 gene, and $91 \%$ and $5 \%$ for detection of the CNV of the pfplasmepsin 2 gene. There was no significant difference in gene copy numbers assessed by uniplex or duplex ddPCR assays regarding CNV in the pfgch1 gene $(p=0.276)$. The accuracy and \%RSD value of the duplex ddPCR assay were $95 \%$ and $4 \%$, respectively, regarding pfgch $1 \mathrm{GCN}$. In the P. falciparum field samples, pfmdr1 and pfplasmepsin2 GCNs were amplified in 15\% and 27\% of samples from Ubon Ratchathani, Thailand, while pfgch 1 GCN was amplified in 50\% of samples from Yala, Thailand. There was 100\% agreement between the GCN results obtained from the $\mathrm{ddPCR}$ and $\mathrm{gPCR}$ assays $(K=1.00)$. The results suggested that multiplex $\mathrm{ddPCR}$ assay is the optional assay for the accurate detection of gene copy number without requiring calibration standards, while the cost and required time are reduced. Based on the results of this study, criteria for GCN detection by ddPCR analysis were generated.
\end{abstract}

\footnotetext{
*Correspondence: noi@tropmedres.ac

${ }^{5}$ Department of Molecular Tropical Medicine and Genetics, Faculty

of Tropical Medicine, Mahidol University, Bangkok, Thailand

Full list of author information is available at the end of the article
}

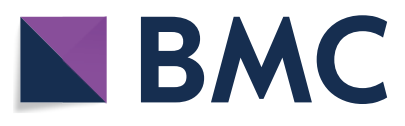

(c) The Author(s) 2021. This article is licensed under a Creative Commons Attribution 4.0 International License, which permits use, sharing, adaptation, distribution and reproduction in any medium or format, as long as you give appropriate credit to the original author(s) and the source, provide a link to the Creative Commons licence, and indicate if changes were made. The images or other third party material in this article are included in the article's Creative Commons licence, unless indicated otherwise in a credit line to the material. If material is not included in the article's Creative Commons licence and your intended use is not permitted by statutory regulation or exceeds the permitted use, you will need to obtain permission directly from the copyright holder. To view a copy of this licence, visit http://creativeco mmons.org/licenses/by/4.0/. The Creative Commons Public Domain Dedication waiver (http://creativecommons.org/publicdomain/ zero/1.0/) applies to the data made available in this article, unless otherwise stated in a credit line to the data. 
Conclusions: The developed ddPCR assays are simple, accurate, precise and cost-effective tools for detection of the CNVs in the pfmdr1, pfplasmepsin2 and pfgch1 genes of P. falciparum. The ddPCR assay is a useful additional tool for the surveillance of anti-malarial drug resistance.

Keywords: ddPCR, Plasmodium falciparum, pfmdr1, pfplasmepsin2, pfgch1

\section{Background}

Artemisinin-based combination therapy (ACT) is recommended as front-line treatment for Plasmodium falciparum malaria, which remains an important infectious disease in tropical regions. However, the emergence and spread of resistance to artemisinin-based combinations and related drugs have resulted in poor curative rates, especially in Southeast Asia [1-5]. Molecular surveillance is needed not only for the detection of mutations to the $P$. falciparum kelch gene, which are associated with artemisinin resistance [3], but also molecular markers associated with the efficacy of other anti-malarial drugs. An increase in the $P$. falciparum multidrug resistance 1 (pfmdr1) GCN is associated with mefloquine resistance [6], while an increase in the P. falciparum plasmepsin2 (pfplasmepsin2) GCN is associated with piperaquine resistance $[7,8]$. Moreover, amplification of the $P$. falciparum GTP cyclohydrolase 1 (pfgch1) GCN is linked to upregulation of the $P$. falciparum dihydrofolate reductase (pfdhfr) and P. falciparum dihydropteroate synthase (pfdhps) genes, which are associated with sulfadoxinepyrimethamine resistance in Southeast Asia $[9,10]$.

Quantitative PCR (qPCR) assays are conventionally used to assess the copy number variations (CNVs) of genes related to drug resistance in P. falciparum malaria (i.e. pfmdr1 [6], pfplasmepsin2 [7], and pfgch1 [11]). Alternatively, droplet digital PCR (ddPCR) technology was developed to measure CNVs and to provide highly precise measurements of the concentrations of target and reference genes in DNA samples [12, 13], as well as tolerance to PCR inhibitors, such as heparin [14], and to generate calibration curves to determine the GCNs of target sequences $[15,16]$. The ddPCR assay is based on water-oil emulsion droplet technology used for detection and quantification of target gene [17]. The ddPCR reaction contains the ddPCR reagent, DNA samples, primers and fluorescent probe. All components are divided into around 15,000-20,000 droplets using the droplet generator. Each droplet may contain one, more than one or no copies of the DNA target $[12,13,18]$. After 40 cycles of the standard PCR reaction, DNA targets in each droplet are amplified and then analysed by a droplet reader. The DNA target concentration is calculated from the number of positive and negative droplets using Poisson statistics [12]. The ddPCR assay has been developed for accurate detection of CNVs in human genes associated with various human genetic diseases [19-21]. In addition, a ddPCR assay was developed and validated for the detection and quantification of Plasmodium species based on the $18 S$ rRNA gene sequence [22, 23], but this method has not yet been validated for the detection of the CNVs of genes associated with resistance to anti-malarial drugs.

In the present study, ddPCR assays were developed and validated for quantification of the CNVs of the pfmdr1, pfplasmepsin 2 and pfgch 1 genes, as well as for the molecular surveillance of the efficacy of anti-malarial drugs in field isolates. The ddPCR assays were used to detect the CNVs of the pfmdr1, pfplasmepsin 2 and pfgch1 genes in field samples and validated against the results obtained with qPCR assays. A flowchart was generated including criteria for the detection of GCN with the novel ddPCR assays. Costs and time required for the ddPCR assays were discussed.

\section{Methods \\ DNA samples}

Development and validation of the ddPCR assays used DNA extracted from $P$. falciparum laboratory strains obtained from the Malaria Research and Reference Reagent Resource Center (American Type Culture Collection, Manassas, VA, USA). Parasite DNA from $P$. falciparum strain 3D7 (MRA-102), which carries single copies of the pfmdr1 and pfplasmespin 2 genes, was used to develop and validate the ddPCR assays for the detection of the CNVs of these two genes. DNA samples of $P$. falciparum strain D6 originating from Sierra Leone, West Africa, which carries a single copy of the pfgch1 gene [10], were used as single copy controls for the development and validation of ddPCR assays to detect the CNVs of the pfgch1 gene. Two-fold serial dilutions of $P$. falciparum strains 3D7 (approximate 40,000 P. falciparum genome copies/ul based on the absolute quantification of pfßtubulin gene) and D6 (approximate 50,000 P. falciparum genome copies/ul based on the absolute quantification of pfßtubulin gene) were prepared and used to quantify the GCN, as well as to assess the accuracy and precision of the ddPCR assays.

Plasmodium falciparum strains 3D7 (isolated in Amsterdam), 7G8 (isolated in Brazil), D6 (isolated in Sierra Leone), D10 (isolated in Papua New Guinea), Dd2 
(derived from cultivation), HB3 (isolated in Honduras), and W2 (isolated in Lao People's Democratic Republic) obtained from the Malaria Research and Reference Reagent Resource Center $(n=7)$ were used to compare the CNVs obtained by the ddPCR and qPCR assays. In addition, the CNVs of the pfmdr1, pfplasmepsin 2 and pfgch 1 genes of field samples $(n=84)$ collected from patients with confirmed P. falciparum infections between 2015 and 2019 in Ubon Ratchathani $(n=60)$ and Yala $(n=24)$, Thailand, were determined. DNA samples were extracted using the QIAamp DNA Mini Kit (Qiagen, Hilden, North Rhine-Westphalia, Germany). DNA concentrations were measured using a Nanodrop ${ }^{\mathrm{TM}}$ spectrophotometer (Thermo Scientific, Willington, DE, USA). The study protocol was approved by the Ethical Review Committee of the Faculty of Tropical Medicine, Mahidol University (Bangkok, Thailand) (approval no. MUTM 2012-045-05).

\section{Development of the ddPCR assays}

For the ddPCR assays, each $20 \mu \mathrm{L}$ reaction contained $10 \mu \mathrm{L}$ of ddPCR ${ }^{\mathrm{TM}}$ Supermix for Probes (Bio-Rad Laboratories, Hercules, CA, USA), $900 \mathrm{nM}$ for each primer $(1.8 \mu \mathrm{L}$ of $10 \mu \mathrm{M}$ of primer), $250 \mathrm{nM}$ for each probe $(0.5 \mu \mathrm{L}$ of $10 \mu \mathrm{M}$ probe) and $2 \mu \mathrm{L}$ of DNA as a template. The primers and probes were previously designed for qPCR assays $[6,7,11]$. The $P$. falciparum $\beta$ tubulin (pfßtubulin) gene was used as reference housekeeping gene. The ddPCR reaction was separated into $12,000-20,000$ droplets using a QX200 ${ }^{\mathrm{TM}}$ Droplet Generator (Bio-Rad Laboratories) and conducted using a $\mathrm{T} 100^{\mathrm{TM}}$ Thermal Cycler (Bio-Rad Laboratories). During development of the ddPCR assays, a series of temperatures was tested to determine the optimal annealing temperature. Uniplex, duplex and multiplex ddPCR assays were developed to measure the CNVs of the pfmdr1, pfplasmepsin 2 and pfgch 1 genes. The optimal annealing temperature for the uniplex ddPCR assay of the pfmdr1, pfplasmepsin2, pfgch1 and pfßtubulin genes was determined to be $56{ }^{\circ} \mathrm{C}$ (Additional file 1), while that for the duplex ddPCR assay for detection of pfmdr1/pfßtubulin and pfplasmepsin $2 / p f \beta t u b u l i n$ genes was $58{ }^{\circ} \mathrm{C}$ and for the duplex ddPCR assay of the pfgch1/pfßtubulin genes, the optimal annealing temperature was $60{ }^{\circ} \mathrm{C}$ (Additional file 2). The optimal annealing temperature for the multiplex ddPCR assay of the pfmdr1/pfplasmepsin2/pfßtubulin genes was $60{ }^{\circ} \mathrm{C}$ (Fig. 1). For validation, the ddPCR assays were performed in triplicate. After amplification, the ddPCR data were read with the use of a QX200 ${ }^{\mathrm{TM}}$ Droplet Reader (Bio-Rad Laboratories) and analysed using QuantaSoft ${ }^{\mathrm{TM}}$ Software version 1.7.4 (Bio-Rad Laboratories). At least 12,000 accepted droplets were analysed $[24,25]$. Manual thresholds were applied to distinguish between positive and negative droplets. The fluorescence intensity thresholds were determined manually for each independent experiment using the using the clustering tool in the QuantaSoft ${ }^{\mathrm{TM}}$ Software. Positive and negative controls were included in each run. The GCN was calculated as the ratio of the concentration (copies $/ \mu \mathrm{L}$ ) of the target gene to that of the reference gene.

\section{Validation of ddPCR assays}

Two-fold serial dilutions of P. falciparum strain 3D7 were prepared to quantify the CNVs of the $p f m d r 1$ and pfplasmepsin 2 genes, as well as validation of the accuracy and precision of the uniplex, duplex and multiplex ddPCR assays. Two-fold serial dilutions of $P$. falciparum strain D6 were prepared for validation of the uniplex and duplex ddPCR assays of the CNV of the pfgch 1 gene. The GCN was determined by three independent ddPCR runs. The accuracy of the ddPCR assays was calculated as \% accuracy $=100-\%$ error and \%error $=$ the absolute difference between 1 and the GCN determined with the ddPCR assays. The precision of the ddPCR assays was calculated as the percent relative standard deviation (\%RSD) = standard deviation/ average $\times 100$. Since the DNA samples might contain both P. falciparum and human DNA, the limit of the $\mathrm{GCN}$, as determined with the ddPCR assays, was quantified based on the lambda $(\lambda)$ value, which was calculated as $\lambda=\ln$ (number of negative droplets/numbers of accepted droplets). The limit of GCN quantification of the ddPCR assays is the range of the $\lambda$ value providing a \%RSD value of greater than $20 \%$ and \%accuracy value of greater than $80 \%[25,26]$. In accordance with the guidelines of the Minimum Information for Publication of Quantitative Digital PCR Experiments [27], the GCN results of the $P$. falciparum reference strain were

(See figure on next page.)

Fig. 1 Two dimensional ddPCR amplitude plots of multiplex ddPCR assays. Heat map shows 8 clusters of droplets (a) including, droplets contain pfmdr1, pfplasmepsin2, and pfbtubulin (cluster 1), droplets contain both pfplasmepsin2 and pf- $\beta$-tubulin (cluster 2), droplets contain both pfmdr1 and pf- $\beta$-tubulin (cluster 3), droplets with at least one copy of pf- $\beta$-tubulin (cluster 4), droplets contain both pfmdr 1 and pf- $\beta$-tubulin (cluster 5), droplets with at least one copy of pfplasmepsin2 (cluster 6), droplets with at least one copy of pfmdr1 (cluster 7), Empty droplets, no DNA target (cluster 8). Classification cluster of droplets for pfmdr1 copy number detection (b). Classification cluster of droplets for pfplasmepsin2 copy number detection (c) 


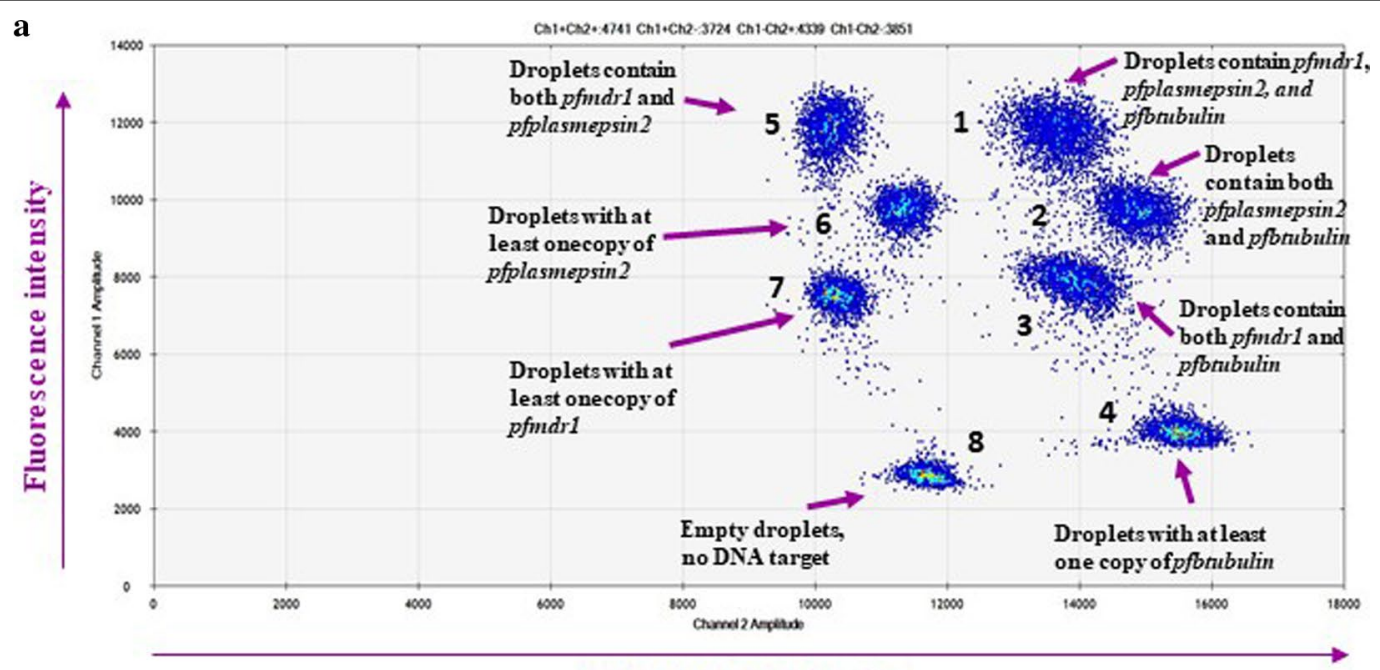

Fluorescence intensity

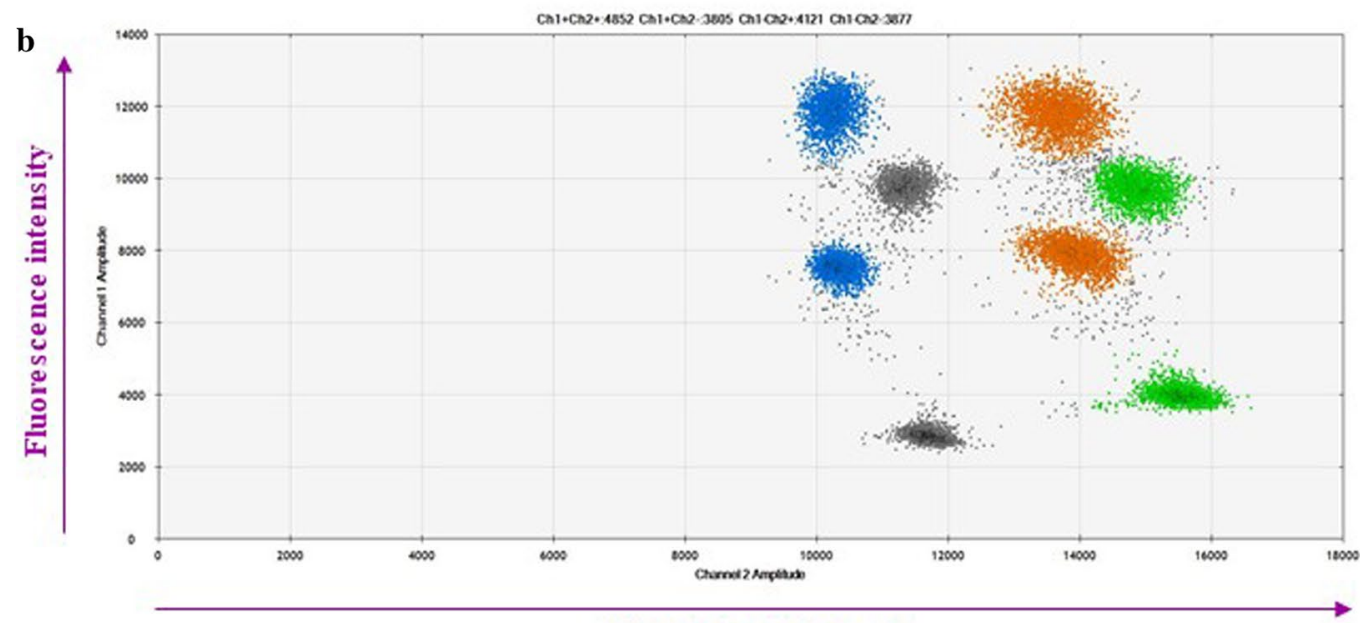

Fluorescence intensity

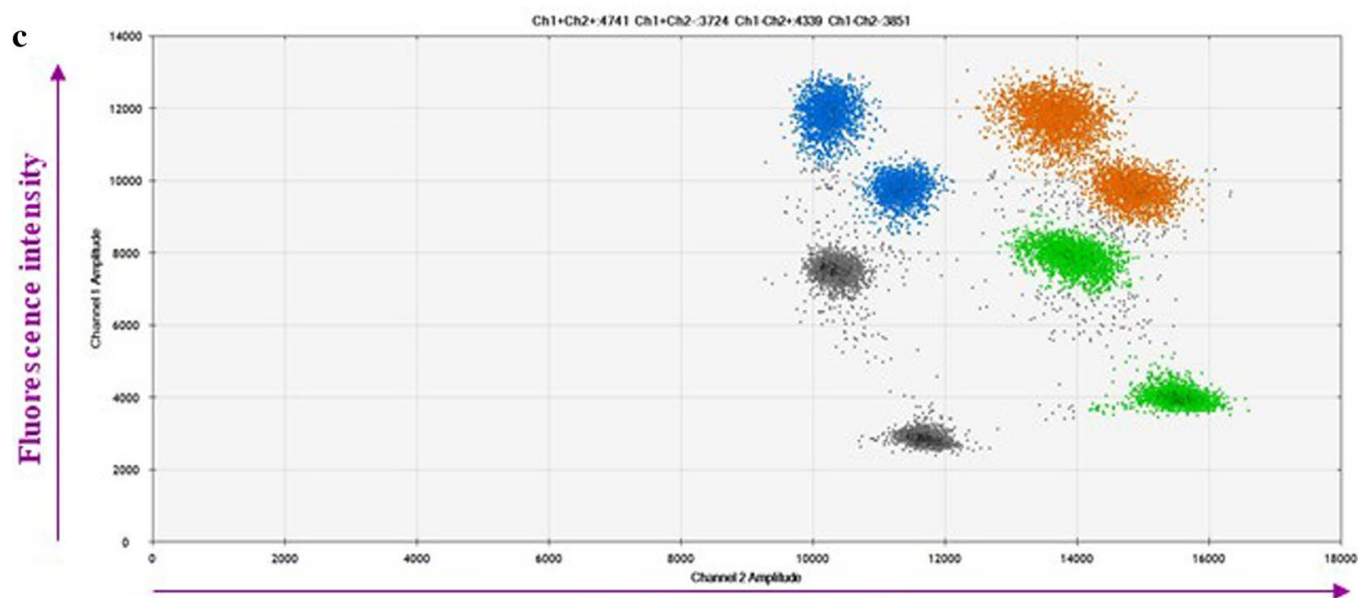

Fluorescence intensity 

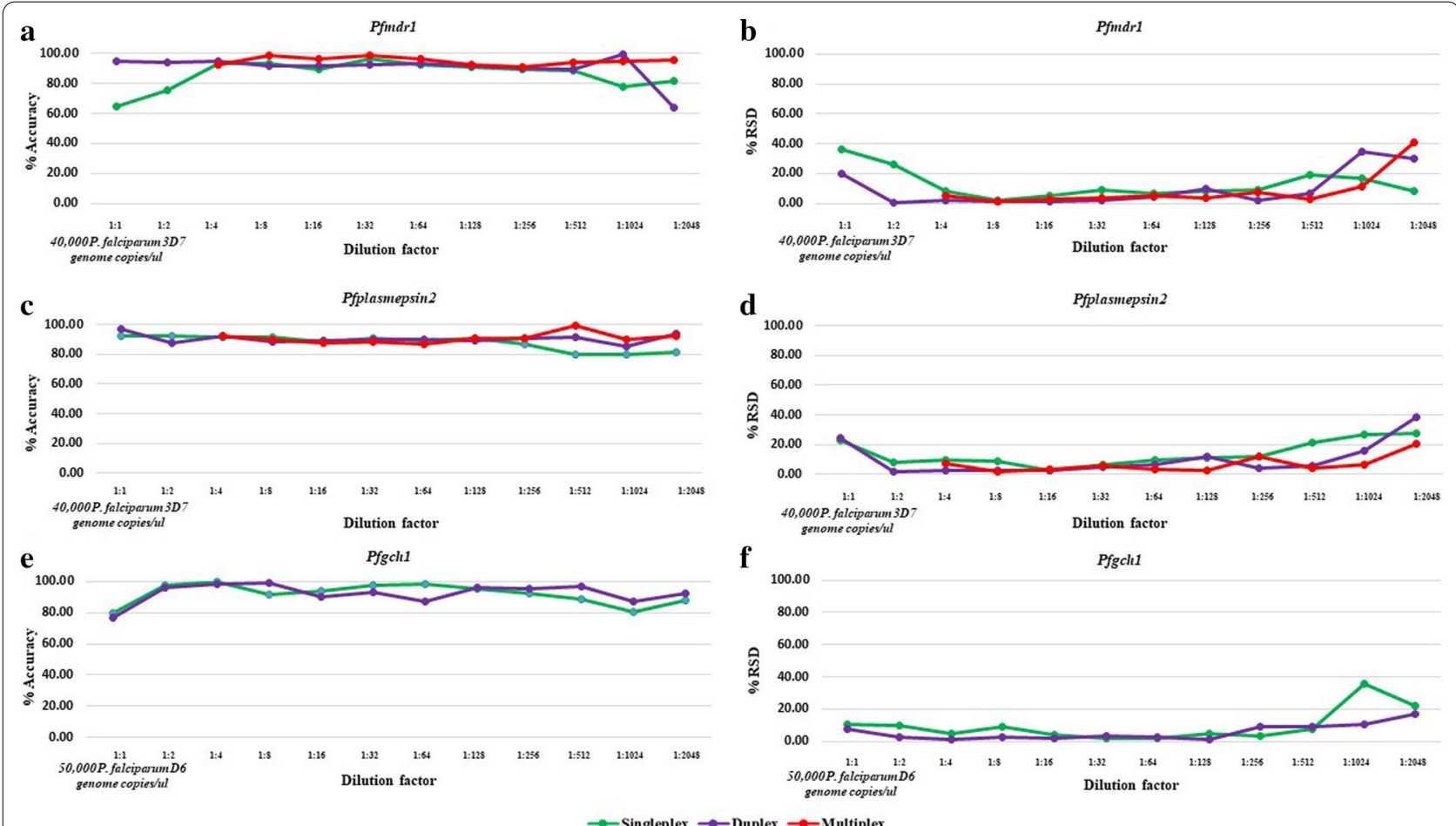

Fig. 2 Limitation of gene quantification, accuracy and precision of ddPCR assays for pfmdr1 copy number detection (a), pfplasmepsin2 copy number detection (b), and pfgch1 copy number detection (c)

compared between the uniplex, duplex and multiplex ddPCR assays.

\section{Assessment of pfmdr1, pfplasmespsin2 and pfgch1 CNVs of $P$. falciparum reference strains and field isolates from Thailand}

DNA samples from the $P$. falciparum reference strain $(\mathrm{n}=7)$ and the $P$. falciparum isolates from Thailand $(n=84)$ were used to evaluate the ddPCR assays. The $\mathrm{CNV}$ results obtained by the ddPCR assays were compared with the results of the GPCR assays, as previously described $[6,7,11]$.

\section{Statistical analysis}

The GCN results of the $P$. falciparum reference strain, as determined with the uniplex, duplex and multiplex ddPCR assays, were compared using the independent samples median test with IBM SPSS Statistics for Windows, version 22.0 (IBM Corporation, Armonk, NY, USA). The kappa statistic was used to identify agreements between the GCN results obtained with the ddPCR assays and those obtained with the qPCR assays with the use of IBM SPSS Statistics for Windows, version 22.0.

\section{Results}

\section{Development and validation of ddPCR assays for CNV} measurements

\section{Accuracy of the ddPCR assays}

As shown in Fig. 2, the accuracies of the uniplex, duplex and multiplex ddPCR assays were 65-96\%, 64-99\% and 91-99\%, respectively, for measurement of the pfmdr1 GCN, and 76-96\%, 85-97\% and 87-99\%, respectively, for measurement of the pfplasmepsin2 GCN. Meanwhile, the accuracies of the uniplex and duplex ddPCR assays for measurement of the pfgch1 GCN were $80-100 \%$ and 77-99\%, respectively.

\section{Precision of ddPCR assays}

As shown in Fig. 2, the \%RSD values of the uniplex, duplex and multiplex ddPCR assays were $2-36 \%, 0-35 \%$ and $1-41 \%$, respectively, for detection of the pfmdr1 GCN, and $3-28 \%, 2-39 \%$ and $2-21 \%$, respectively, for detection of the pfplasmepsin2 GCN. Meanwhile, the \%RSD values of the uniplex and duplex ddPCR assays were $2-36 \%$ and $1-17 \%$ for detection of the $p f g c h 1$ GCN.

\section{Limitation of GCN quantification}

As shown in Table 1, the accepted range of $\lambda$ values of the uniplex, duplex and multiplex ddPCR assays were $0.011-0.987,0.005-2.178$ and $0.003-0.842$, respectively, 
Table 1 Descriptive statistics of $P$. falciparum mdr1, plasmepsin2, and gch1 CNVs based on the accepted lamda( $\lambda$ ) value of ddPCR assay of $p f-\beta$-tubulin gene

\begin{tabular}{|c|c|c|c|c|c|c|c|c|}
\hline \multirow[t]{2}{*}{ Statistic } & \multicolumn{3}{|l|}{ pfmdr1 CNVs } & \multicolumn{3}{|c|}{ pfplasmepsin2 CNVs } & \multicolumn{2}{|l|}{ pfgch1 CNVs } \\
\hline & Singleplex & Duplex & Multiplex & Singleplex & Duplex & Multiplex & Singleplex & Duplex \\
\hline Range & 0.253 & 0.177 & 0.225 & 0.315 & 0.276 & 0.214 & 0.324 & 0.236 \\
\hline Minimum & 0.812 & 0.817 & 0.861 & 0.814 & 0.738 & 0.813 & 0.868 & 0.926 \\
\hline Maximum & 1.065 & 0.994 & 1.086 & 1.129 & 1.014 & 1.027 & 1.192 & 1.162 \\
\hline Mean & 0.922 & 0.919 & 0.964 & 0.943 & 0.895 & 0.906 & 1.016 & 1.039 \\
\hline SD & 0.061 & 0.040 & 0.061 & 0.085 & 0.057 & 0.057 & 0.078 & 0.065 \\
\hline Variance & 0.004 & 0.002 & 0.004 & 0.007 & 0.003 & 0.003 & 0.006 & 0.004 \\
\hline$\%$ RSD & 6.954 & 3.273 & 4.755 & 8.675 & 5.947 & 5.385 & 5.282 & 3.705 \\
\hline \%Accuracy & 92.240 & 92.135 & 95.047 & 93.304 & 89.533 & 90.638 & 95.118 & 94.876 \\
\hline $\begin{array}{l}\text { Accepted } \\
\text { lamda }(\lambda) \\
\text { range }\end{array}$ & $0.011-0.976$ & $0.005-2.173$ & $0.003-0.839$ & $0.010-1.860$ & $0.002-1.890$ & $0.003-0.938$ & $0.006-1.909$ & $0.003-1.874$ \\
\hline
\end{tabular}

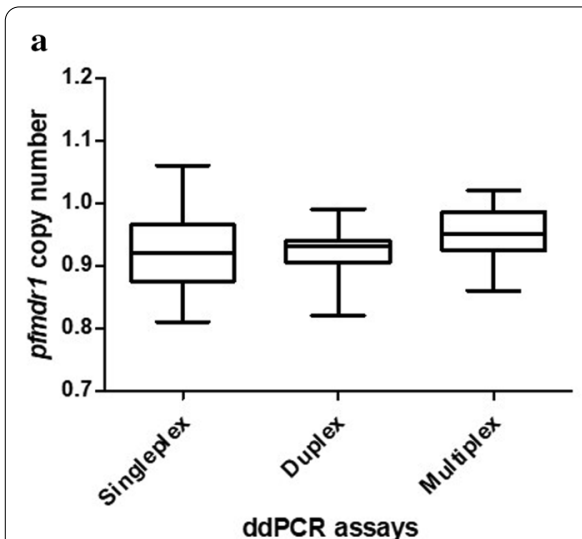

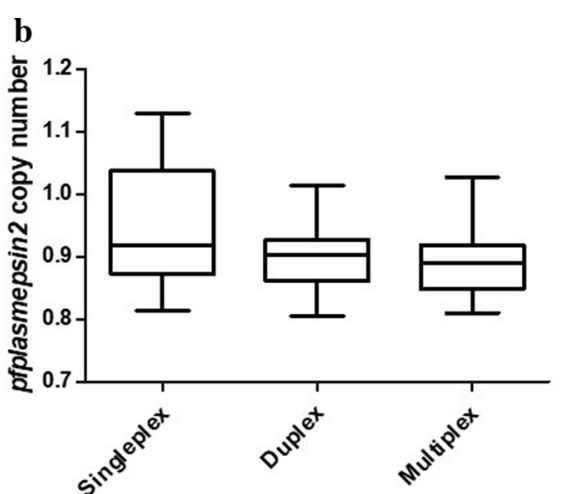

ddPCR assays

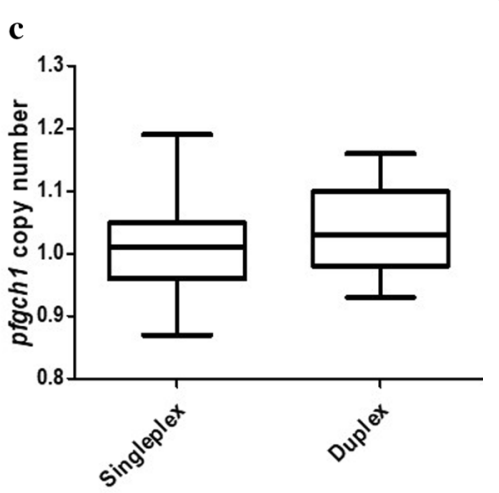

ddPCR assays

Fig. 3 Whisker plots show median, maximum, and minimum of estimated pfmdr1 (a), pfplasmepsin2 (b), and pfgch1 (c) copy number

for the pfmdr1 GCN, and $0.010-1.870,0.002-1.890$ and $0.003-0.941$, respectively, for the pfplasmepsin2 GCN. The accepted range of $\lambda$ values of the uniplex and duplex ddPCR assays were $0.006-1.915$ and $0.003-1.877$, respectively, for the pfgch1 GCN. Based on the limitation of GCN quantification, the average accuracy and \%RSD value of the multiplex ddPCR assay were $95 \%$ and $5 \%$, respectively, for measurement of the pfmdr $1 \mathrm{GCN}$, and $91 \%$ and $5 \%$, respectively, for measurement of the pfplasmepsin 2 GCN. The accuracy and \%RSD value of the duplex ddPCR were $95 \%$ and $4 \%$, respectively.

\section{Comparison between the uniplex, duplex and multiplex ddPCR assays}

Two-fold serial dilutions of DNA from $P$. falciparum strain 3D7 (4, 2, 1, 0.5, 0.25, 0.125, 0.0625, 0.03125, $0.015625,0.0078125,0.00390625$ and $0.001953125 \mathrm{ng} /$ $\mu \mathrm{L}$ ) were used to compare the CNVs of the pfmdr1 and pfplasmepsin 2 genes obtained from the uniplex, duplex and multiplex ddPCR assays. In addition, two-fold serial dilutions of DNA from $P$. falciparum strain D6 $(8,4,2$, $1,0.5,0.25,0.125,0.0625,0.03125,0.015625,0.0078125$ and $0.00390625 \mathrm{ng} / \mu \mathrm{L}$ ) were used for comparison of the CNVs of the pfgch 1 gene obtained with the uniplex and duplex ddPCR assays. As shown in Fig. 3, there were no significant differences in the pfmdrl $(p=0.363)$ and pfplasmepsin $2(p=0.330)$ GCNs, as determined with the uniplex, duplex and multiplex ddPCR assays. In addition, there was no significant difference for detection of the $p f g c h 1 \mathrm{GCN}$ between the uniplex and duplex ddPCR assays $(p=0.276)$.

\section{Standardized analytical workflow of ddPCR analysis for quantification of GCN}

A flowchart, including validation criteria, for a standardized analytical workflow of ddPCR analysis was designed based on the observed limit of quantification of the optimal accuracy and precision. Control samples, as well as 
positive and negative controls, were included for each ddPCR assay. The accepted criteria used for the ddPCR assay are that the results of the negative control are negative, those of the positive single copy control are positive (ratio $=0.80-1.20$ ) and those of the positive multiple copy control are positive (ratio $>1.20$ ). Each ddPCR reaction contained at least 12,000 droplets. The GCN results were considered acceptable at a $\lambda$ value of $0.003-0.800$ for quantification of the pfmdr 1 and pfplasmepsin 2 genes with the multiplex ddPCR assay, and 0.003-1.900 for quantification of the $p f g c h 1$ gene with the duplex ddPCR assay. GCNs were calculated as the ratio of the concentrations (copies $/ \mu \mathrm{L}$ ) of the target and references genes (single GCN ratio of $0.8-1.2$ and multiple GCN ratio of $>1.20$ ).

\section{Agreement of GCN results between the ddPCR and qPCR assays}

DNA samples from the $P$. falciparum reference strain $(\mathrm{n}=7)$ and $P$. falciparum strains 3D7, 7G8, D10, DD2, HB3, W2 and D6 were collected to compare the pfmdr1, pfplasmepsin 2 and pfgch 1 GCNs determined with the ddPCR and qPCR assays. The results showed 100\% agreement between the ddPCR and qPCR assays $(\kappa=1)$ (Fig. 4, Additional file 3).

\section{CNVs of the pfmdr1, pfplasmespsin2 and pfgch1 genes of $P$. falciparum isolates from Thailand}

The extracted DNA samples were determined the CNVs in the pfmdr1, pfplasmespsin 2 and pfgch 1 genes following the standardized analytical workflow obtained from this study. The results showed that the $p f m d r 1$ and pfplasmepsin 2 GCNs were amplified in $15 \%$ and $27 \%$ of samples from Ubon Ratchathani, Northeastern Thailand, suggested evidence of selection whereas no amplification in isolates from Yala, Southern Thailand. For the pfgch $1 \mathrm{GCN}$ was amplified in 50\% of samples from Yala, while no amplification in isolates from Ubon Ratchathani (Fig. 5, Additional file 4). Comparisons of the results of the $\mathrm{ddPCR}$ and $\mathrm{qPCR}$ assays were $100 \%$ in agreement for CNV assessments of the pfmdr1, pfplasmepsin2 and pfgch1 genes.

\section{Cost and turn-around time of ddPCR assays}

The costs of the uniplex, duplex and multiplex ddPCR assays to determine the CNVs of the pfmdr1, pfplasmepsin 2 and pfgch 1 genes were 10.40, 5.50 and 5.70 USD per sample, respectively. The turn-around times for the uniplex, duplex and multiplex ddPCR assays of 96 samples were12, 6 and 6 h, respectively.

\section{Discussion}

The ddPCR has been suggested as the reliable alternative method for high-throughput GCNs quantification [12, 13]. Previously, ddPCR assay was developed for screening gene deletions and duplications in human genes such as Breast cancer type 1 susceptibility protein (BRCA1) which plays a significant role in carcinogenesis of breast and ovarian cancer [20], Leucine-rich repeats and immunoglobulin-like domains 1 (LRIG1) which may be determinants of breast cancer prognostic marker [28], and mitochondrial DNA (mtDNA) which varies during aging and disease progression [29]. In malaria, qPCR assay is the most commonly used assay for the identification of genes associated with anti-malarial drug resistance $[6,7$, 11]. Here, ddPCR assays were developed and validated for accurate assessment of the CNVs of several $P$. falciparum resistance genes. GCNs estimated by the qPCR assay were measured based on exponential curves and calculated by the formula; ( $\mathrm{Ct}$ of a target gene $-\mathrm{Ct}$ of a reference gene) of sample - (Ct of a target gene $-\mathrm{Ct}$ of a reference gene) of reference sample or $=2^{-\Delta \Delta \mathrm{Ct}}$, while estimate with the ddPCR assays were measured based on the ratio of the absolute gene concentrations of the target and reference genes [13]. Therefore, GCNs of ddPCR were measured without requiring the reference sample.

Although, the ddPCR assay was suggested for accurate GCNs quantification, previous study demonstrated that the accuracy and precision of GCNs quantification were reduced when using too high or too low concentration of the target genes [30]. Similarly, the results of the present study demonstrated that a higher or lower concentration of the target gene might affect the accuracy and precision of the ddPCR assays. As a consequence, optimal concentrations of the target genes are required for accurate detection of the GCNs. Here, an optimal DNA template was evaluated to accurately and precisely determine the GCN based on the $\lambda$ value, which is estimated from the numbers of negative and accepted droplets generated by the ddPCR assays. So, the limit of quantification of the multiplex ddPCR assay of pfmdr 1 and pfplasmepsin 2 were based on the $\lambda$ value which is between 0.003 and 0.8 and the limit of quantification of the duplex ddPCR assay is also based on the $\lambda$ value which is between 0.003 and 1.9 for duplex ddPCR assay (Fig. 6).

To reduce the cost and turn-around time, multiplex ddPCR assays were developed for detection of both the pfmdr 1 and pfplasmepsin 2 genes in a single reaction. The results showed that there were no significant differences in the GCN assessments between the assays, favouring the multiplex, rather than the uniplex, ddPCR assay as the preferred method [27]. In, addition, a duplex ddPCR assay was also developed for the detection of the pfgch1 GCN instead of the uniplex ddPCR assay. Although there 
a

pfindr1 gene copy number estimate

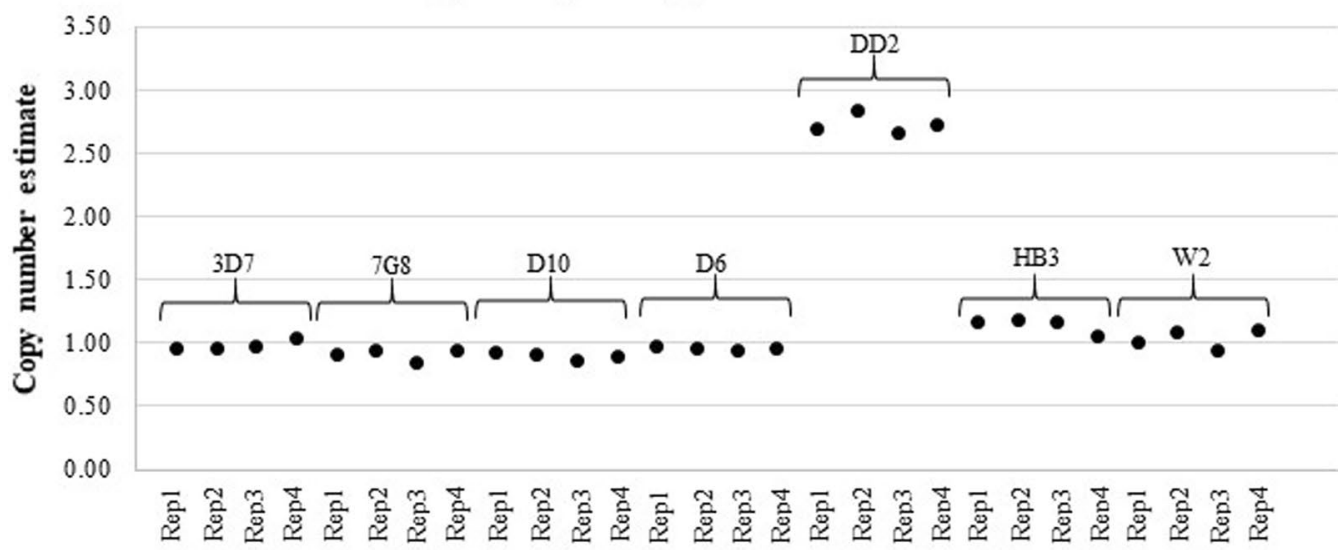

Samples replicates

b

pfplasmepsin 2 gene copy number estimate
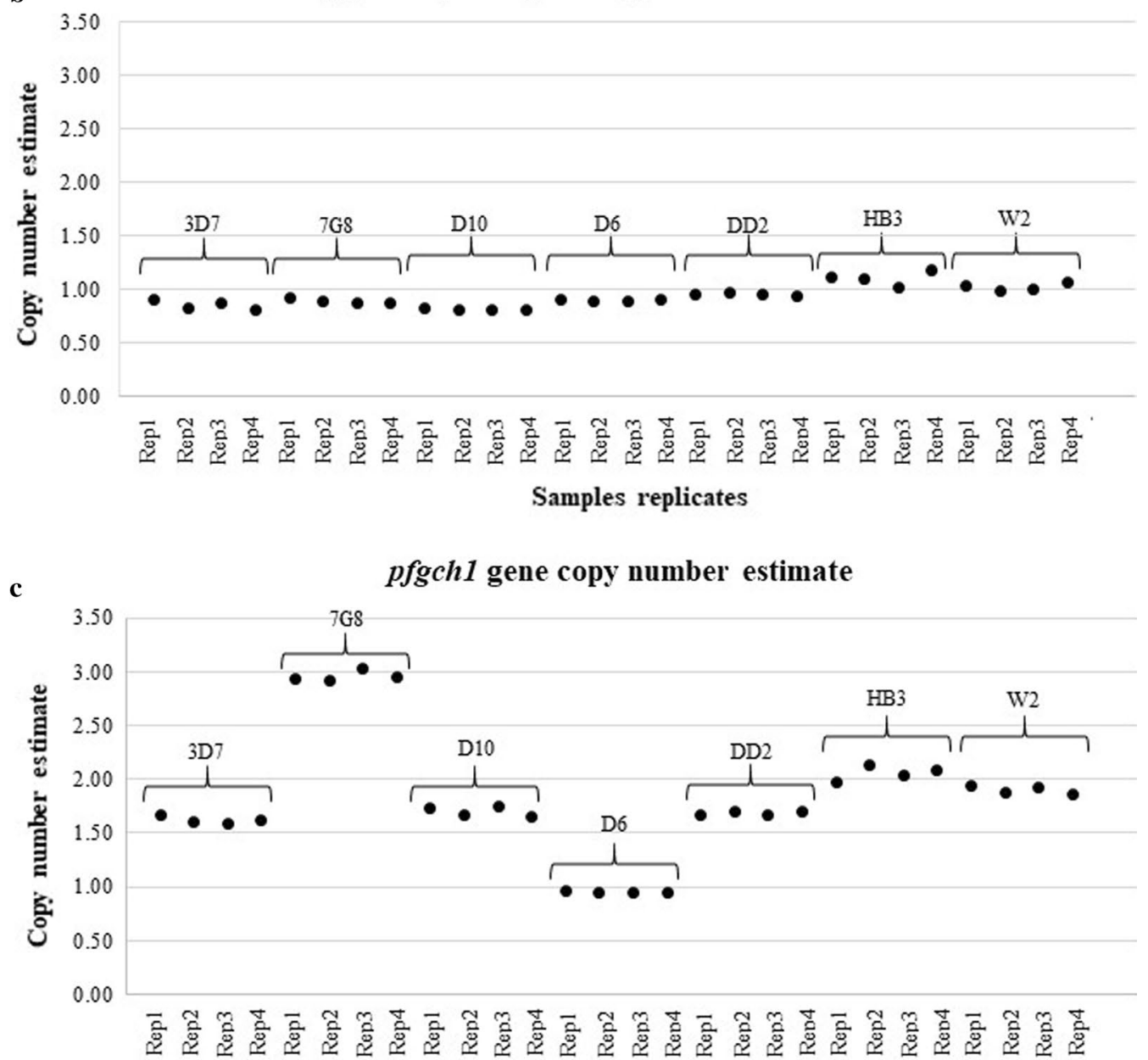

Samples replicates

Fig. 4 Genes copy number of P. falciparum $m d r 1$ (a), plasmepsin2 (b), and gch1 of reference strains estimated by ddPCR in replicates 


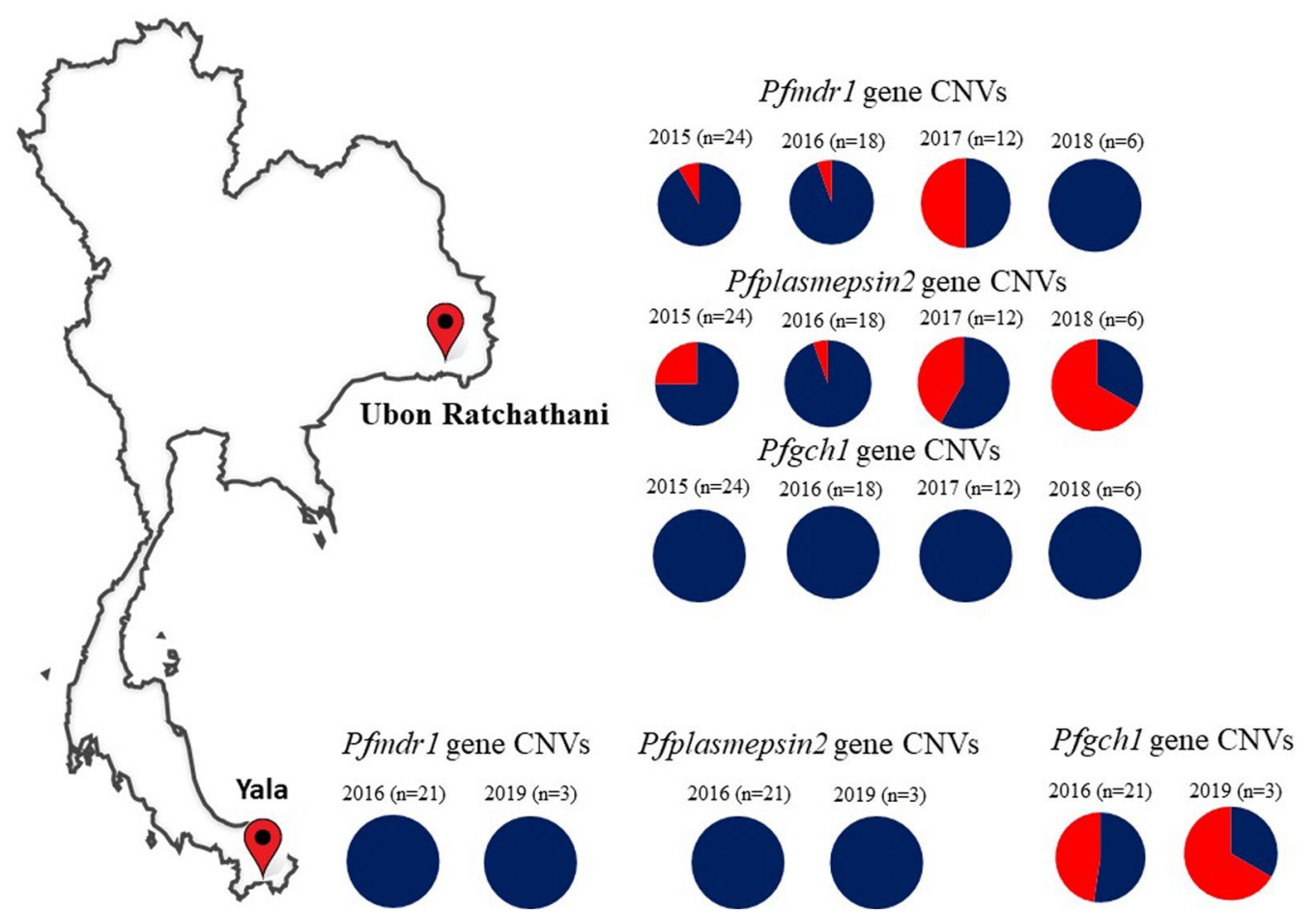

Fig. 5 Prevalence of pfmdr1, pfplasmepsin2, and pfgch1 gene amplification isolated from Ubon Ratchathani and Yala

was no significant difference between the GCNs results obtained from the singleplex and multiplex ddPCR assay, for the culture strain experiments, the $p f m d r 1$ and pfplasmepsin 2 copy number relative to $p f \beta t u b u l i n$ ratio is consistently below 1.0 with medians near 0.9 for all suggested that the results obtained from multiplex ddPCR would be further normalized with a number to provide more accurate value.

Compared to the uniplex ddPCR assay, use of the duplex ddPCR assay can reduce costs by $47 \%$ from 10.40 to 5.47 USD, while the estimated cost of qPCR is around 6.7 USD per sample. Duplex ddPCR assay can reduce the required assay time by $50 \%$ from 12 to $6 \mathrm{~h}$. Moreover, the use of the multiplex ddPCR PCR assay to detect the pfmdr 1 and pfplasmepsin 2 GCNs reduced costs by $72 \%$ from 20.80 to 5.73 USD and reduced the required time by $75 \%$ from 24 to $6 \mathrm{~h}$.

The prevalence of CNVs of the pfmdrl, pfplasmepsin2, which are the molecular markers of mefloquine, piperaquine resistance respectively, and pfgch1 linked to $p f d h f r / p f d h p s$ mutations which resulted in sulfadoxine-pyrimethamine resistance were assessed in Thai samples collected from year 2015-2019. The GCN results obtained with the ddPCR and qPCR assays were in $100 \%$ agreement. The proportion of isolates with amplified $p f m d r 1$ remains at low prevalence or zero in the two locations. This could be results of low drug pressure as mefloquine was discontinued as a national policy for treatment of uncomplicated falciparum malaria in Thailand since 2013. Since then piperaquine has been replaced [31]. The results of pfplasmepsin2 gene amplification associated with piperaquine resistance were in agreement with previous publications [32, 33], which showed amplification of pfplasmepsin 2 in Northeast Thailand from 2011 to 2018. This suggested piperaquine resistance in $P$. falciparum is prevalent in Northeast Thailand.

Although sulfadoxine-pyrimethamine (SP) antimalarial treatment was no longer used as a national policy for treatment of uncomplicated falciparum malaria in Thailand from since 1990, the results of the present study revealed substantial amplification of the pfgch1 in Yala, near the Malaysian border. Persistence of high prevalence of antifolate resistance haplotypes in Thailand may be explained by several factors, such as continued drug pressure from non-malarial antifolate drugs such as trimethoprim and sulfamethoxazole [34], therefore, antifolate gene mutations/amplification might have been sustained because of continued presence of this antifolate drug pressure. The prevalence of the $p f m d r 1$, pfplasmepsin 2 and pfgch 1 GCNs obtained from this study might be useful for surveillance of the efficacy of anti-malarial drugs. 
A standardized analytical workflow of ddPCR analysis for genes copy number quantification.

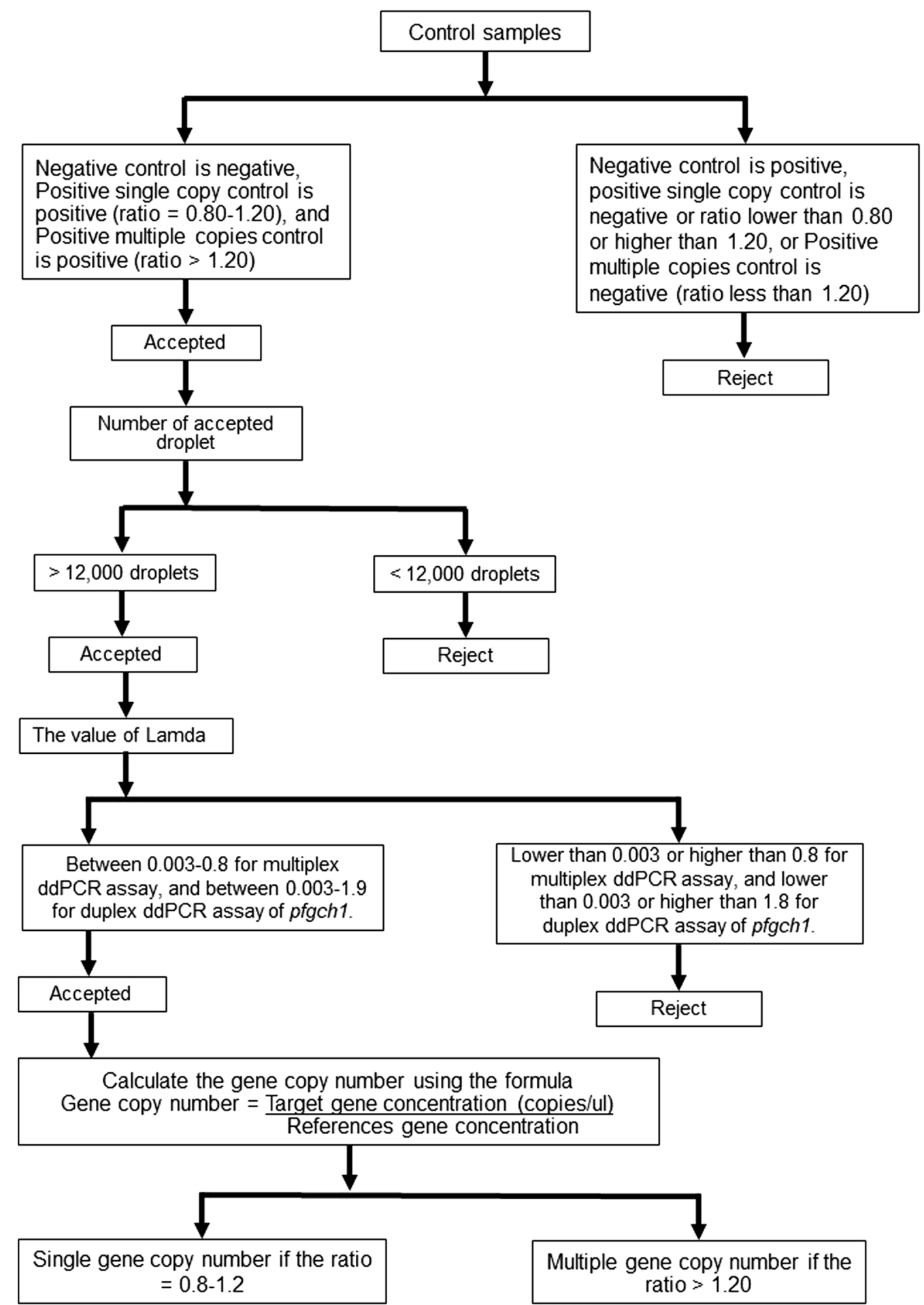

Fig. 6 A standardized analytical workflow of ddPCR analysis used for genes copy number quantification 


\section{Conclusions}

Uniplex, duplex and multiplex ddPCR assays for detection of the CNVs of the P. falciparum $m d r 1$, plasmepsin 2 and $g c h 1$ genes were developed and validated. The results confirmed the accuracy and precision of the proposed assays, which reduced the cost and turnaround time for surveillance of the efficacy of antimalarial drugs. The assay is a valuable additional tool for genetic surveillance of anti-malarial drug resistance.

\section{Author details}

${ }^{1}$ Department of Clinical Microscopy, Faculty of Allied Health Sciences, Chulalongkorn University, Bangkok, Thailand. ${ }^{2}$ Mahidol-Oxford Tropical Medicine Research Unit, Faculty of Tropical Medicine, Mahidol University, Bangkok, Thailand. ${ }^{3}$ Division of Vector Borne Diseases, Department of Disease Control, Ministry of Public Health, Nonthaburi, Thailand. ${ }^{4}$ Centre for Tropical Medicine and Global Health, Nuffield Department of Medicine, University of Oxford, Oxford, UK. ${ }^{5}$ Department of Molecular Tropical Medicine and Genetics, Faculty of Tropical Medicine, Mahidol University, Bangkok, Thailand.

Received: 2 November 2020 Accepted: 19 February 2021

Published online: 28 February 2021

\section{Supplementary Information}

The online version contains supplementary material available at https://doi. org/10.1186/s12936-021-03659-5.

Additional file 1. Primers and probes used for ddPCR and qPCR assays, and the optimal annealing temperature used.

Additional file 2. Two dimensional ddPCR amplitude plots of duplex $d d P C R$ assays. The duplex ddPCR assay of pfmdr1/pf- $\beta$-tubulin plot (a.), duplex ddPCR assay of pfplasmepsin $2 / p f-\beta$-tubulin plot (b.), and duplex $\mathrm{ddPCR}$ assay of pfgch1/pf- $\beta$-tubulin plot (c.) shows droplets with at least one copy of target genes (blue), reference gene (green), droplets contain both target and reference gene (orange), and empty droplets (grey).

Additional file 3. Genes copy number of $P$. falciparum reference strains estimated by ddPCR and qPCR assays.

Additional file 4. Genes copy number of P. falciparum isolated from Thailand.

\section{Abbreviations}

DNA: Deoxyribonucleic acid; ddPCR: Droplet digital PCR; GCN: Gene copy number; PCR : Polymerase chain reaction; $18 \mathrm{~S}$ rRNA : 18s ribosomal RNA gene; pfmdr1: P. falciparum multidrug resistance 1 gene; pfgch 1: P. falciparum GTP cyclohydrolase 1 gene; pfplasmepsin2: P. falciparum plasmepsin2 gene; nM: Nanomolar; $\mu \mathrm{L}$ : Microliter.

\section{Acknowledgements}

We would like to thank Ms. Jureeporn Duanguppama, Mr. Chanon Kunason, Ms. Watcharee Pagornrat, and Ms. Wanassanan Madmanee for their help.

\section{Authors' contributions}

SS and MI contributed to study design. RS collected samples. SS and KS undertook laboratory work. SS and MI analysed data. SS, AD and MI drafted the manuscript. All authors read and approved the final manuscript.

\section{Funding}

This study was supported by Thailand Science Research and Innovation (TSRI) and National research council of Thailand, RTA6280006 and Faculty of Tropical Medicine, Mahidol University Thailand and the Wellcome Trust Mahidol University Oxford Tropical Medicine Research Programme.

\section{Availability of data and materials}

All data generated or analyzed during this study are included in this published article and its supplementary information files.

\section{Ethics approval and consent to participate}

Ethical approvals for the study were obtained from the ethical review committees of the Faculty of Tropical Medicine, Mahidol University (MUTM 2012-04505). Informed consent was obtained from all participants.

\section{Consent for publication}

Not applicable.

\section{Competing interests}

The authors declare that they have no competing interests.

\section{References}

1. Dondorp AM, Nosten F, Yi P, Das D, Phyo AP, Tarning J, et al. Artemisinin resistance in Plasmodium falciparum malaria. N Engl J Med. 2009;361:455-67.

2. Phyo AP, Nkhoma S, Stepniewska K, Ashley EA, Nair S, McGready R, et al. Emergence of artemisinin-resistant malaria on the western border of Thailand: a longitudinal study. Lancet. 2012;379:1960-6.

3. Ashley EA, Dhorda M, Fairhurst RM, Amaratunga C, Lim P, Suon S, et al. Spread of artemisinin resistance in Plasmodium falciparum malaria. N Engl J Med. 2014;371:411-23.

4. Denis MB, Tsuyuoka R, Poravuth Y, Narann TS, Seila S, Lim C, et al. Surveillance of the efficacy of artesunate and mefloquine combination for the treatment of uncomplicated falciparum malaria in Cambodia. Trop Med Int Health. 2006;11:1360-6.

5. Amaratunga C, Lim P, Suon S, Sreng S, Mao S, Sopha C, et al. Dihydroartemisinin-piperaquine resistance in Plasmodium falciparum malaria in Cambodia: a multisite prospective cohort study. Lancet Infect Dis. 2016;16:357-65.

6. Price RN, Uhlemann AC, Brockman A, McGready R, Ashley E, Phaipun $L$, et al. Mefloquine resistance in Plasmodium falciparum and increased pfmdr1 gene copy number. Lancet. 2004;364:438-47.

7. Witkowski B, Duru V, Khim N, Ross LS, Saintpierre B, Beghain J, et al. A surrogate marker of piperaquine-resistant Plasmodium falciparum malaria: a phenotype-genotype association study. Lancet Infect Dis. 2017;17:174-83.

8. Amato R, Lim P, Miotto O, Amaratunga C, Dek D, Pearson RD, et al. Genetic markers associated with dihydroartemisinin-piperaquine failure in Plasmodium falciparum malaria in Cambodia: a genotype-phenotype association study. Lancet Infect Dis. 2017;17:164-73.

9. Hamour S, Melaku Y, Keus K, Wambugu J, Atkin S, Montgomery J,et al. Malaria in the Nuba Mountains of Sudan: baseline genotypic resistance and efficacy of the artesunate plus sulfadoxine-pyrimethamine and artesunate plus amodiaquine combinations. Trans R Soc Trop Med Hyg. 2005;99:548-54.

10. Kidgell C, Volkman SK, Daily J, Borevitz JO, Plouffe D, Zhou Y, et al. A systematic map of genetic variation in Plasmodium falciparum. PLoS Pathog. 2006;2:e57.

11. Nair S, Miller B, Barends M, Jaidee A, Patel J, Mayxay M, et al. Adaptive copy number evolution in malaria parasites. PLoS Genet. 2008:4:e1000243.

12. Hindson BJ, Ness KD, Masquelier DA, Belgrader P, Heredia NJ, Makarewicz AJ, et al. High-throughput droplet digital PCR system for absolute quantitation of DNA copy number. Anal Chem. 2011;83:8604-10.

13. Pinheiro LB, Coleman VA, Hindson CM, Herrmann J, Hindson BJ, Bhat S, et al. Evaluation of a droplet digital polymerase chain reaction format for DNA copy number quantification. Anal Chem. 2012;84:1003-11.

14. Dingle TC, Sedlak RH, Cook L, Jerome KR. Tolerance of droplet-digital $P C R$ vs real-time quantitative PCR to inhibitory substances. Clin Chem. 2013:59:1670-2.

15. Bhat S, Curach N, Mostyn T, Bains GS, Griffiths KR, Emslie KR. Comparison of methods for accurate quantification of DNA mass concentration with traceability to the international system of units. Anal Chem. 2010:82:7185-92.

16. Campomenosi P, Gini E, Noonan DM, Poli A, D'Antona P, Rotolo N, et al. Imperatori A: A comparison between quantitative PCR and droplet digital 
PCR technologies for circulating microRNA quantification in human lung cancer. BMC Biotechnol. 2016;16:60.

17. Vogelstein B, Kinzler KW. Digital PCR. Proc Natl Acad Sci USA. 1999;96:9236-41.

18. Nakano M, Komatsu J, Matsuura S, Takashima K, Katsura S, Mizuno A. Single-molecule PCR using water-in-oil emulsion. J Biotechnol. 2003;102:117-24.

19. Ito T, Kawashima Y, Fujikawa T, Honda K, Makabe A, Kitamura K, et al. Rapid screening of copy number variations in STRC by droplet digital PCR in patients with mild-to-moderate hearing loss. Hum Genome Var. 2019;6:41.

20. Oscorbin I, Kechin A, Boyarskikh U, Filipenko M. Multiplex ddPCR assay for screening copy number variations in BRCA1 gene. Breast Cancer Res Treat. 2019;178:545-55.

21. Harmala SK, Butcher R, Roberts CH. Copy number variation analysis by droplet digital PCR. Methods Mol Biol. 2017;1654:135-49.

22. Koepfli C, Nguitragool W, Hofmann NE, Robinson LJ, Ome-Kaius M, Sattabongkot J, et al. Sensitive and accurate quantification of human malaria parasites using droplet digital PCR (ddPCR). Sci Rep. 2016;6:39183.

23. Srisutham S, Saralamba N, Malleret B, Renia L, Dondorp AM, Imwong M. Four human Plasmodium species quantification using droplet digital PCR. PLOS ONE. 2017;12:e0175771.

24. Hughesman CB, Lu XJ, Liu KY, Zhu Y, Poh CF, Haynes C. A robust protocol for using multiplexed droplet digital PCR to quantify somatic copy number alterations in clinical tissue specimens. PLoS One. 2016;11:e0161274.

25. Ludlow AT, Shelton D, Wright WE, Shay JW. ddTRAP: a method for sensitive and precise quantification of telomerase activity. Methods Mol Biol. 2018:1768:513-29.

26. Bhat S, Herrmann J, Armishaw P, Corbisier P, Emslie KR. Single molecule detection in nanofluidic digital array enables accurate measurement of DNA copy number. Anal Bioanal Chem. 2009;394:457-67.

27. Huggett JF, Foy CA, Benes V, Emslie K, Garson JA, Haynes R, et al. The digital MIQE guidelines: minimum information for publication of quantitative digital PCR experiments. Clin Chem. 2013;59:892-902.
28. Faraz M, Tellstrom A, Ardnor CE, Grankvist K, Huminiecki L, Tavelin B, et al. LRIG1 gene copy number analysis by ddPCR and correlations to clinical factors in breast cancer. BMC Cancer. 2020;20:459.

29. Memon AA, Zoller B, Hedelius A, Wang X, Stenman E, Sundquist J, et al. Quantification of mitochondrial DNA copy number in suspected cancer patients by a well optimized ddPCR method. Biomol Detect Quantif. 2017;13:32-9.

30. Deprez L, Corbisier P, Kortekaas AM, Mazoua S, Beaz Hidalgo R, Trapmann $\mathrm{S}$, et al. Validation of a digital PCR method for quantification of DNA copy number concentrations by using a certified reference material. Biomol Detect Quantif. 2016;9:29-39.

31. WHO. Country cooperation strategy, Thailand: 2017-2021. Geneva, World Health Organization. 2017.

32. van der Pluijm RW, Imwong M, Chau NH, Hoa NT, Thuy-Nhien NT, Thanh $N V$, et al. Determinants of dihydroartemisinin-piperaquine treatment failure in Plasmodium falciparum malaria in Cambodia, Thailand, and Vietnam: a prospective clinical, pharmacological, and genetic study. Lancet Infect Dis. 2019;19:952-61.

33. Imwong M, Dhorda M, Myo Tun K, Thu AM, Phyo AP, Proux S, et al. Molecular epidemiology of resistance to antimalarial drugs in the Greater Mekong subregion: an observational study. Lancet Infect Dis. 2020;20:1470-80.

34. Iyer JK, Milhous WK, Cortese JF, Kublin JG, Plowe CV. Plasmodium falciparum cross-resistance between trimethoprim and pyrimethamine. Lancet. 2001;358:1066-7.

\section{Publisher's Note}

Springer Nature remains neutral with regard to jurisdictional claims in published maps and institutional affiliations.
Ready to submit your research? Choose BMC and benefit from:

- fast, convenient online submission

- thorough peer review by experienced researchers in your field

- rapid publication on acceptance

- support for research data, including large and complex data types

- gold Open Access which fosters wider collaboration and increased citations

- maximum visibility for your research: over $100 \mathrm{M}$ website views per year

At BMC, research is always in progress.

Learn more biomedcentral.com/submissions 